\title{
COMORBILIDADES ASOCIADAS A INFECCIÓN DE TRACTO URINARIO POR ESCHERICHIA COLI BLEE POSITIVO DEL HOSPITAL VITARTE. $2017-2018$
}

\author{
COMORBIDITIES ASSOCIATED WITH EXTENDED- SPECTRUM BETA-LACTAMASE ESCHERICHIA COLI \\ URINARY TRACT INFECTION, VITARTE HOSPITAL. 2017 -2018
}

\author{
Yanina Chipa-Paucar ${ }^{1,2, a}$
}

\begin{abstract}
RESUMEN
Introducción: Las infecciones por bacterias productoras de $\beta$-lactamasas de espectro extendido (BLEE) son un serio problema en nuestro país desde principios del año 2000 se ha dado un aumento progresivo en la frecuencia de las infecciones causadas por productoras de $\beta$-lactamasas de espectro extendido (BLEE). Objetivo: Determinar las comorbilidades asociadas a pacientes con Infección del Tracto Urinario causada por E. coli BLEE del servicio de medicina interna en el Hospital de Vitarte en el periodo 2017-2018. Métodos: Estudio observacional, analítico de tipo casos y controles. Se contó con una muestra correspondiente a 114 divididos en 57 casos y 57 controles. Se recolectó la información mediante la revisión de historias clínicas y el uso de una ficha de recolección de datos. Se utilizó estadística descriptiva con medidas de tendencia central y dispersión, en el análisis bivariado la regresión logística, y en el análisis multivariado modelos lineales generalizados. Resultados: La mediana de la edad de la población fue de 66,5 RI: 19-97 años, siendo de sexo femenino el $79,82 \%(n=23)$ y de raza mestiza el $46,49 \%(n=53)$. En referencia a los que tuvieron $E$. Coli BLEE, su edad tuvo una mediana de 64 años con RI: 19-97, y el 43,48\% ( $n=10)$ del sexo masculino la tuvo al igual que el $63,64 \%(n=4)$ de los que tuvieron obstrucción urinaria, $55,56 \%(n=5)$ de los que tuvieron incontinencia urinaria, $61,02 \%$ $(n=36)$ de los que tuvieron diabetes mellitus, $65 \%(n=13)$ de los obesos, $57,14 \%(n=32)$ de los hipertensos y $62,71 \%$ ( $\mathrm{n}=32$ ) de los que tuvieron ITU recurrente. Se determinó en el análisis bivariado que DM $(\mathrm{OR}: 2,53, \mathrm{IC}: 1,19-5,39, \mathrm{p}=0,016)$ e ITU recurrente (OR:2,94, IC:1,37-6,3, $p=0,005)$ fueron significativos. En el análisis multivariado aquellos que tuvieron ITU recurrente tuvieron 1.61 veces la probabilidad de tener ITU E. Coli BLEE que los que no tuvieron ITU recurrente con IC:1,07-2,43, p=0,022 ajustado por diabetes mellitus. Conclusión: La diabetes mellitus y la ITU recurrente fueron comorbilidades independientemente asociadas a la ITU E. coli BLEE, siendo la ITU recurrente la comorbilidad asociada de forma multivariada ajustada por diabetes mellitus.
\end{abstract}

Palabras clave: Infección Tracto Urinario; Escherichia coli; BLEE; Diabetes Mellitus. (fuente: DeCS BIREME)

\begin{abstract}
Introduction: The infections by bacteria producing b-lactamasas extended spectrum (ESBL) are a serious problem in our country since the beginning of the year 2000 there has been a progressive increase in the frequency of infections caused by these bacteria, especially Escherichia coli and Klebsiella spp. Objective: To determine the comorbidities associated with patients with Urinary Tract Infection caused by e. colli BLEE of the Internal Medicine Service in the Vitarte Hospital in the period 2017-2018. Methods: Observational, case-control study. There was a sample corresponding to 114 divided into 57 cases and 57 controls. The information was collected through the review of medical records and the use of a data collection form. Descriptive statistics were used with measures of central tendency and dispersion, in the bivariate analysis the logistic regression, and in the multivariate analysis generalized linear models. Results: The median age of the population was $66,5 \mathrm{RI}$ : $19-97$ years, being $79,82 \%$ female $(n=23)$ and $46,49 \%$ mestizo $(n=$ 53). In reference to those who had E. Coli BLEE, their age had a median of 64 years with RI: 19-97, and $43,48 \%$ ( $n=$ $10)$ of male sex had it as did $63,64 \%(n=4))$ of those who had urinary obstruction, $55,56 \%(n=5)$ of those who had urinary incontinence, $61,02 \%(n=36)$ of those who had diabetes mellitus, $65 \%(n=13)$ of the obese, $57,14 \%(n=32)$ of the hypertensive patients and $62,71 \%(n=32)$ of those who had recurrent UTI. It was determined in the bivariate analysis that DM (OR: 2,53, Cl: 1,19-5,39, $p=0,016)$ and recurrent UTI (OR: 2,94, CI: 1,37-6,3, $\mathrm{p}=0,005)$ were significant. In the multivariate analysis those who had recurrent UTI had 1,61 times the probability of having UTI of E. coli ESBL than those who did not have recurrent UTI with HF: 1,07-2,43, $p=0,022$ adjusted for diabetes mellitus. Conclusion: Diabetes mellitus and recurrent UTI were comorbidities independently associated with UTI E. Coli BLEE, with recurrent UTI associated multivariate comorbidity adjusted for diabetes mellitus.
\end{abstract}

Key words: Urinary tract infection; Escherichia coli; EEBL; Diabetes Mellitus. (source: MeSH NLM)

\footnotetext{
1 Hospital de vitarte-Minsa,Lima-Perú

2 Facultad de Medicina Humana, Universidad Ricardo Palma,Lima-Perú.

a Médico cirujano.

Citar como: Yanina Chipa-Paucar. Comorbilidades asociadas a infección de tracto urinario por Escherichia Coli Blee positivo del hospital vitarte. 2017 - 2018. Rev. Fac. Med. Hum. Julio 2019; 19(3):48-52. DOI 10.25176/RFMH.v19i3.2162

Journal home page: http://revistas.urp.edu.pe/index.php/RFMH

Artículo publicado por la Revista de la Facultad de Medicina Humana de la Universidad Ricardo Palma. Es un artículo de acceso abierto, distribuído bajo los términos de la Licencia Creative Commons: Creative Commons Attribution 4.0 International, CC BY 4.0 (https://creativecommons.org/licenses/by/4.0/), que permite el uso no comercial, distribución y reproducción en cualquier medio, siempre que la obra original sea debidamente citada. Para uso comercial, por favor póngase en contacto con revista.medicina@urp.pe
} 


\section{INTRODUCCIÓN}

Las infecciones del tracto urinario (ITU), son consideradas a nivel mundial como una de los motivos más frecuentes de consultas médicas1, presentando según la Organización Mundial de la Salud (OMS) una incidencia de 2-3 casos por cada 100 mil habitantes por año generando altos costos y carga para los sistemas de salud a nivel mundial2. En el $90 \%$ de ITUs, el agente etiológico relacionado es la Escherichia coli, sin embargo, esto no excluye que exista una amplia gama de patógenos relacionados3. En la actualidad la automedicacion es un problema de salud pública que tiene un efecto directo sobre la resistencia bacteriana aunado a las comorbilidades propias del paciente que pueden deprimir su sistema inmunológico haciéndolo mucho más susceptible a plural. Por ejemplo, el uso indiscriminado de cefalosporinas de tercera generación trajo como consecuencia, primero en Europayluegoen el mundo, a lasbacterias productoras de betalactamasa de espectro extendido ( BLEE), cuya participación en las infecciones va en aumento según diferentes reportes en Latinoamérica4.

\section{MÉTODOS}

Estudio observacional, analítico transversal. Como criterios de selección se tuvo para la inclusión de los casos a pacientes con ITU y E. coli BLEE positivo confirmado con cultivo, e historia clínica completa. En los controles se tuvo, a los E. Coli BLEE negativo. Como criterios de exclusión se tuvo a pacientes embarazadas, menores de 18 años, urocultivos polimicrobianos e ITU nosocomial. Se contó con una muestra correspondiente a 114 divididos en 57 ITU BLEE y 57 ITU no BLEE. Se recolectó la información mediante la revisión de historias clínicas a partir de un muestreo aleatorizado y el uso de una ficha de recolección de datos. Se utilizó estadística descriptiva con medidas de tendencia central y dispersión. En el análisis bivariado la regresión logística, y en el análisis multivariado modelos lineales generalizados.

Para el análisis bivariado se analizaron las diferencias entre categorías siendo el caso que aquellas cuyas pruebas estadísticas dieran como resultados $p<0,05$ serían significativas y por lo tanto, indicarían que existen diferencias entre categorías y éstas se deben al azar. Se utilizaron para variables cuantitativas las pruebas de Tde student (Normal) yU Mann Whitney (No normal) según el tipo de distribución.
Para las variables cualitativas, se utilizaron las pruebas de chi2 y Fisher según los valores esperados.

En referencia al análisis multivariado, se utilizaron modelos lineales generalizados con distribución binomial y función logística para analizar la ITU BLEE positiva y la DM e ITU recurrente.

\section{RESULTADOS}

Se contó con historias clínicas de 114 pacientes, divididos en 57 expuestos con ITU E. coli BLEE y 57 no expuestos con ITU E. coli sensible. La mediana de la edad fue de 66 años con rango intercuartílico de 19 a 97 años. El sexo femenino representó un $79,82 \%$ ( $n=91$ ) y la raza mestiza $46,49 \%$ $(n=53)$.

La tabla 1 muestra la descripción de cada variable en torno a la variable dependiente $\mathrm{E}$. coli sensible BLEE positiva. Sea el caso, aquellas que tuvieron ITU sensible a E. coli BLEE tuvieron los siguientes datos: una edad de 64 años con RI: 19,97, el 51,65\%(n=47) fueron mujeres, el 53,83\% ( $n=28)$ de los mestizos, el 48,54\% ( $n=50)$ de los que no tuvieron obstrucción urinaria, el $49,52 \%$ $(n=52)$ de los que no tuvieron incontinencia urinaria, el $61,02 \%(n=36)$ de los que tuvieron diabetes mellitus, el $46,81 \%(n=44)$ de los que no tuvieron obesidad, el $57,14 \%$ ( $n=32)$ de los que tuvieron hipertensión arterial (HTA) y el $62,71 \%(n=37)$ de los que tuvieron ITU recurrente. Para datos adicionales, revisar la tabla 1.

Sólo resultaron significativas las variables diabetes mellitus e ITU recurrente con $p=0,015$ y $p=0,005$ respectivamente. Utilizando la regresión logística se obtuvo que: aquellos que tuvieron diabetes tuvieron 2,53 veces la probabilidad de tener ITU E. coli BLEE en comparación con los que no tuvieron diabetes (IC: 1,19-5,39, p=0,016), y aquellos que tuvieron ITU recurrente tuvieron 2,94 veces la probabilidad de tener ITU E. coli sensible en comparación con los que no tuvieron ITU recurrente (IC: $1,37-6,3, p=0,005)$. Al ser significativas, ambas variables fueron analizas de forma multivariada. Ver Tabla 2.

Se encontró que la ITU recurrente está asociada a ITU BLEE positiva: aquellos que tuvieron ITU recurrente tuvieron 1,61 veces la probabilidad de tener ITU E. coli sensible (IC: 1,07-2.43, p=0,022) que los que no tuvieron ITU recurrente. Ver tabla 3. 
Tabla 1. Características de las variables según E. coli sensible BLEE positivo.

\begin{tabular}{|c|c|c|c|}
\hline \multicolumn{4}{|c|}{ E. coli sensible } \\
\hline & No Blee & Si Blee & $\mathbf{p}$ \\
\hline Edad & 69 RI: 19,93 & 64 RI: 19-97 & 0,2133 \\
\hline \multicolumn{4}{|l|}{ Sexo } \\
\hline Femenino & $44(48,35)$ & $47(51,65)$ & \\
\hline Masculino & $13(56,52)$ & $10(43,48)$ & \\
\hline Raza & & & 0,755 \\
\hline Negra & $13(56,52)$ & $10(43,48)$ & \\
\hline Blanca & $19(50)$ & $19(50)$ & \\
\hline Mestiza & $25(47,17)$ & $28(52,83)$ & \\
\hline Obstrucción urinaria & & & 0,341 \\
\hline No & $53(51,46)$ & $50(48,54)$ & \\
\hline $\mathrm{Si}$ & $4(36,36)$ & $7(63,64)$ & \\
\hline \multicolumn{4}{|l|}{ Incontinencia urinaria } \\
\hline No & $53(50,48)$ & $52(49,52)$ & \\
\hline $\mathrm{Si}$ & $4(44,44)$ & $5(55,56)$ & \\
\hline Diabetes mellitus & & & 0,015 \\
\hline No & $34(61,82)$ & $21(38,18)$ & \\
\hline $\mathrm{Si}$ & $23(38,98)$ & $36(61,02)$ & \\
\hline Obesidad & & & 0,14 \\
\hline No & $50(53,19)$ & $44(46,81)$ & \\
\hline $\mathrm{Si}$ & 7 (35) & $13(65)$ & \\
\hline Hipertensión arterial & & & 0,134 \\
\hline No & $33(56,9)$ & $25(43,1)$ & \\
\hline $\mathrm{Si}$ & $24(42,86)$ & $32(57,14)$ & \\
\hline ITU recurrente & & & 0,005 \\
\hline No & $35(63,64)$ & $20(36,36)$ & \\
\hline Si & $22(37,29)$ & $37(62,71)$ & \\
\hline IMC & 25,01 DS: 3,94 & 25,45 DS:4,42 & $0,577 \mathrm{PA}$ \\
\hline PA Sistólica & 120 RI: 85-184 & $130 \mathrm{RI}: 100-170$ & 0,413 \\
\hline PA Diastólica & $70 \mathrm{RI}: 50-100$ & 80 RI: 51-100 & 0,531 \\
\hline
\end{tabular}

Tabla 2. Análisis bivariado de la ITU E. coli sensible BLEE positiva.

\begin{tabular}{lccc} 
& & ITU e. Coli Sensible & p \\
& RPc & IC & 0,016 \\
Diabetes mellitus & 2,53 & $1,19-5,39$ & 0,005 \\
\hline ITU recurrente & 2,94 & $1,37-6,3$ & \\
\hline
\end{tabular}

Tabla 3. Análisis multivariado de la ITU E. coli sensible BLEE positiva.

\begin{tabular}{lccc} 
& & ITU e. Coli Sensible & p \\
& Rpa & IC & 0,055 \\
Diabetes mellitus & 1,47 & $0,99-2,19$ & 0,022 \\
ITU recurrente & 1,61 & $1,07-2,43$ & \\
\hline
\end{tabular}




\section{DISCUSIÓN}

Las infecciones del tracto urinario son una de las patologias con mayor prevalencia6, siendo la segunda causa de infección extrahospitalaria y la primera causa de infección nosocomial7. En el presente estudio se evidencia que la mayor frecuencia de ITU fue del sexo femenino, en un porcentaje de $79,82 \%(n=91)$. Contrasta la frecuencia de sexo femenino encontrada en este estudio, con la frecuencia en el Hospital Nacional Dos de Mayo Perú en el 2015, donde la frecuencia de ITU por bacterias BLEE, es más del $60 \% 8$. En otro estudio en España también encuentra a la población de sexo femenino frecuente para ITUs por agentes BLEE. En un estudio realizado en Chile, donde se estudia pacientes con diabetes mellitus, se encuentra que la mayor frecuencia es en mujeres (52\%)9. Esta frecuencia en mujeres se debe a la presencia de microorganismo, que llegan por vía ascendente, donde se demuestra que los microorganismos se desplazan hacia la uretra (siendo esta la vía más común que lleva a una infección urinaria); cuyo agente principal es la E. coli4.

Dentro de las comorbilidades más frecuentes, se encontró la Diabetes mellitus, presentándose en mayor proporción los cultivos por e. coli BLEE positivo, con un porcentaje de $61,02 \%$ ( $n=36$ ); lo cual contrasta con un estudio en el Hospital de San José en el año $2017^{10}$. Otro estudio en el Hospital Augusto Hernández Mendoza, señala como una de las comorbilidades frecuentes la diabetes mellitus en un $63 \%{ }^{11}$. Respecto a ITU recurrente en frecuencia con 62.71\%. En un estudio en Tacna - Perú en el 2016 se encontró como factor de riesgo el tener ITU previa o recurrente $(52 \%)^{4}$, el presente estudio de los pacientes con cultivo e coli BLEE positivo con ITU recurrente en frecuencia con $62,71 \%$ y en el estudio realizado en el hogar Clínica San Juan de Dios - 2016, se observó entre los factores de riesgo más frecuentes ITU previa5. Asimismo, en un estudio realizado en Chile sobre los factores de riesgo asociados a ITU, se encontró que la comorbilidad más frecuente era la ITU recurrente estando en un 30,99.

Con respecto a la edad, en este estudio la edad promedio de presentación de las ITUs por E. coli BLEE es 64 años, en concordancia con dos estudios realizados en Perú; en el Hospital Nacional de la Policía (Lima) ${ }^{12}$ y el Hospital Augusto Hernández Mendoza (Ica), donde el grupo etario afectado era mayor de 65 años ${ }^{11}$.

\section{CONCLUSIÓN}

Existen comorbilidades asociadas a pacientes con infección del Tracto Urinario causada por E. coli BLEE positivo del Servicio de Medicina Interna el en Hospital deVitarteenelperíodo2017-2018:laDiabetesMellitus y la ITU recurrente están asociadas. No se encontró asociación para obesidad ni hipertensión arterial.

Contribuciones de autoría: La autora participó en la generación, redacción, recolección de datos y aprobación final del artículo.

Financiamiento: Autofinanciado.

Conflicto de interés: La autora declara no tener conflicto de interés en la publicación de este artículo.

Recibido: 30 de abril 2019

Aprobado: 31 de mayo 2019

Correspondencia: Yanina Nataly Chipa-Paucar

Dirección: Av. Alfredo Benavides 5440, Santiago de Surco 15039, Lima-Perú. Teléfono: (01) 7080000

Correo: yanis_125@hotmail.com 


\section{REFERENCIAS BIBLIOGRÁFICAS}

1. Iñaguazo Montero, Robert Kevin. Factores de riesgo en la Infección del Tracto Urinario causados por e. coli productora de beta lactamasas de especctro extendido. [Ecuador]: Universidad Técnica de Machala;2017.

2. Calle A, Colqui K, Rivera D. Factores asociados a la presentación de Infecciones Urinarias por e. coli productoras de BLEE en el año 2016 en el Hospital Cayetano Heredia, Lima-Perú [Internet]. [Lima]: Universidad Peruana Cayetano Heredia; 2016 [citado 5 de enero de 2019]. Disponible en: http://repositorio.upch.edu.pe/handle/upch/604

3. Blanco V, Maya J, Correa A, Perenguez M, Muñoz JS, Motoa G, et al Prevalencia y factores de riesgo para infecciones del tracto urinario de inicio en la comunidad causadas por Escherichia coli productor de betalactamasas de espectro extendido en Colombia. Enfermedades Infecc Microbiol Clínica [Internet]. noviembre de 2016 [citado 6 de enero de 2019];34(9):559-65. Disponible en: https://www.ncbi.nlm.nih. gov/pmc/articles/PMC5061630/

4. Hotuya Conde, Betsaida Gximena. Factores asociados a infecciones urinarias intrahospitalarias producidas por bacterias productoras de betalactamasas de espectro extendido en adultos mayores del Hospital II Luis Negreiros Vega durante el 2015 [Internet]. [Tacna]: Univ. Nac. Jorge Basadre Grohmann; 2016 [citado 5 de enero de 2019]. Disponible en: http://repositorio.unjbg.edu.pe/handle/UNJBG/2218

5. Cardenas GM. Factores de riesgo asociados a infección del tracto urinario BLEE positivo en pacientes hospitalizados en el Servicio de Medicina del Hogar Clínica San Juan de Dios durante el período EneroOctubre 2015 [Internet]. [Lima]: Universidad Ricardo Palma; 2016 [citado 5 de enero de 2019]. Disponible en: http://repositorio.urp.edu. pe/handle/urp/466?show=full

6. Jiménez-Guerra G, Heras-Cañas $V$, Béjar Molina L del C, SorlózanoPuerto A, Navarro-Marí JM, Gutiérrez-Fernández J. Escherichia coli y Klebsiella pneumoniae productores de betalactamasa de espectro extendido en infecciones de vías urinarias: evolución de la resistencia antibiótica y opciones terapéuticas. Med. Clínica [Internet]. abril de 2018 [citado 6 de enero de 2019];150(7):262-5. Disponible en: https:// linkinghub.elsevier.com/retrieve/pii/S0025775317306516
7. Tovar H, Barragán B, Sprockel J. Infección del tracto urinario en pacientes hospitalizados con diabetes tipo 2. Rev Chil Endocrino Diabetes [Internet]. 2016 [citado 5 de enero de 2019];9(1):6-10. Disponible en: http://www.revistasoched.cl/1_2016/2.pdf

8. Sandoval Pérez Jorge. Factores de riesgo asociados a Infección del Tracto Urinario por Bacilos gram negativos BLEE adquiridos en la comunidad atendidos en el Hospital Nacional Dos de Mayo [Internet]. [Lima]: Universidad Ricardo Palma; [citado 5 de enero de 2019]. Disponible en: http://cybertesis.urp.edu.pe/bitstream/urp/956/1/ Sandoval\%20P\%C3\%A9rez\%20Jorge\%20Jean_2017.pdf

9. Avilés C, Betancour P, Velasco CL, Godoy R. Factores asociados a infecciones urinarias producidas por enterobacterias productoras de beta lactamasas de espectro extendido: una cohorte prospectiva. Rev Chil Infectol [Internet]. 2016;33(6):628-

34. Disponible en: https://scielo.conicyt.cl/scielo.php?script=sci_ arttext\&pid=S0716-10182016000600004

10. Ruiz J. Factores clínicos y epidemiológicos asociados a infecciones del tracto urinario por bacterias BLEE, Hospital San José 2014-2015 [Internet]. [Lima]: Universidad Ricardo Palma; 2017 [citado 5 de enero de 2019]. Disponible en: http://cybertesis.urp.edu.pe/bitstream/ urp/900/1/Ruiz\%20Paredes\%20Jose\%20Ivan\%20Aarnaldo_2017.pdf

11. Arias P. Prevalencia de las Infecciones del Tracto Urinario por enterobacterias productoras de BLEE de la comunidad en adultos en el Hospital Augusto Hernández Mendoza durante el período de enero a junio del año 2017, Ica-Perú [Internet]. [Lima]: Universidad Privada San Juan Bautista; 2018 [citado 5 de enero de 2019]. Disponible en: http:// repositorio.upsjb.edu.pe/handle/upsjb/1594

12. Morote E. Prevalencia de E. Coli BLEE en pacientes mujeres de Hospital Nacional PNP-LNS [Internet]. [Lima]: Universidad Ricardo Palma; 2015 [citado 5 de enero de 2019]. Disponible en: http:// repositorio.urp.edu.pe/handle/urp/565

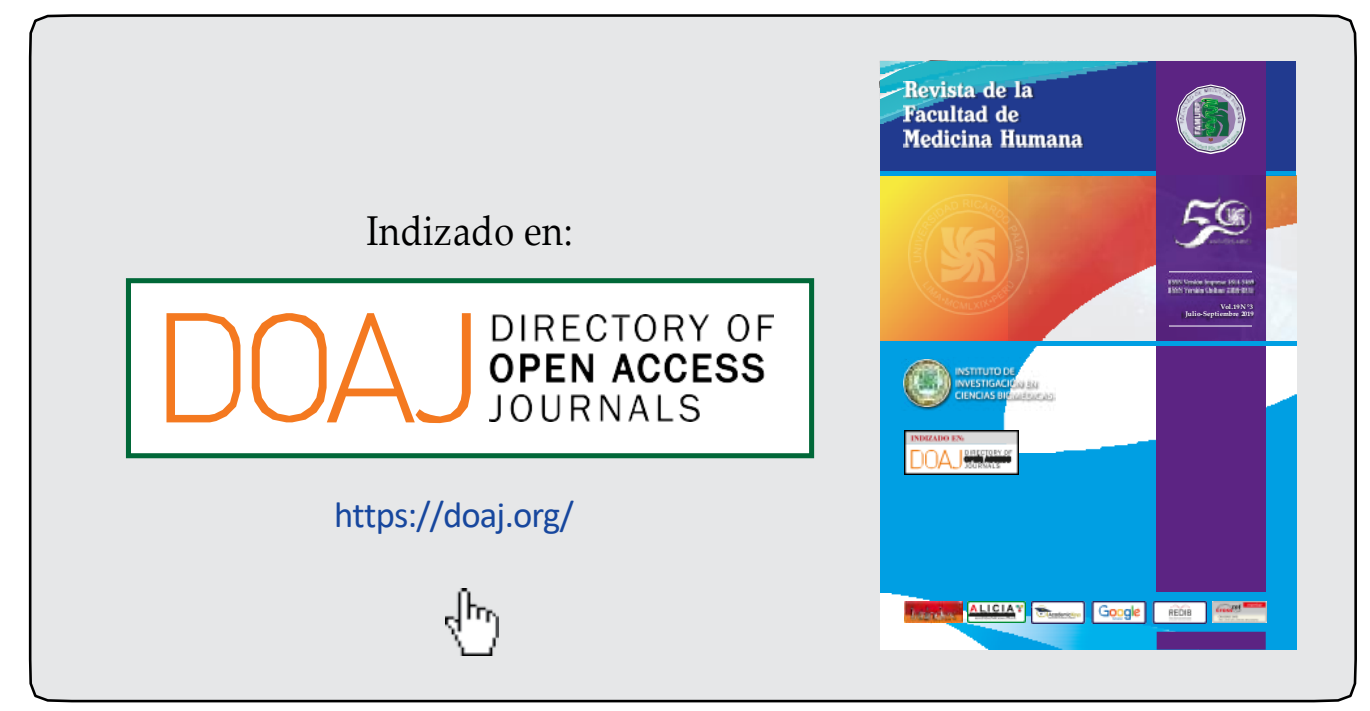

Pág. 52 